\title{
Die Sprachenvielfalt und die Medienstrategien in Algerien
}

\author{
Aoussine Seddiki \\ Sektion Germanistik, Abteilung für angelsächsische Sprachen, Fakultät für Literatur, Sprachen und Künste - \\ Universität Oran, BP 1524, El Menouer, 31.000 Oran, Algérie \\ E-mail: aouseddiki@yahoo.fr
}

\begin{abstract}
The audio-visual media represents one of the main vehicles of internationalisation of our time. Such internationalisation is encouraged by the use of English, as well as by a specific focus on various ethnic groups in the development of media and programs that topicalise language. In various countries and different regions of the world, particular importance is attached to a number of aspects of cultural and linguistic variation within the framework of processing information. Experts try to use international languages, particularly English, French, Arabic, Spanish, German, and Chinese, for the purpose of reaching the masses. At the same time, there is special interest in the African languages (Swahili, Luo, Urdu, Wolof, Berber, and Afrikaans, for example) in the respective areas. In Algeria this strategy towards internationalisation is adopted as well modes of the media, such as television, broadcasting, newspapers and the internet.
\end{abstract}

Keywords: language variety, media strategy, print media, radio, television

Schlüsselbegriffe: Sprachenvielfalt, Medienstrategie, Printmedien, Radio, Fernseh

\section{Einleitung}

Im vorliegenden Beitrag gehe ich der Frage der Vielfalt der Medien nach, mit der wir es heute zu tun haben. Gleichzeitig untersuche ich den Aspekt der Mehrsprachigkeit in den Medien. In Algerien, einem nordafrikanischen und mehrsprachigen Land sind die Menschen mit dem Phänomen des Sprachpluralismus im Alltag bestens vertraut, nicht zuletzt im Hinblick auf Medien und Nachrichten. Mit großem Erstaunen stellen die ausländischen Gäste bei uns fest, wie flexibel und spontan die Einheimischen in ihrem Alltag von einer Sprache zu einer anderen (algerische Variante des Arabischen, Hocharabisch, Berberisch, Französisch) wechseln. In der algerischen Gesellschaft ist das Nebeneinander von Dialekt und Hochsprachen auch in den Medien präsent: Die Printmedien sowie die Radio- und Fernsehprogramme in Algerien nutzen die stark verbreiteten Sprachen und Dialekte. Auf diese Erscheinung, die für die algerische Presse charakteristisch ist, werde ich an späterer Stelle meines Beitrags zu sprechen kommen. Ich möchte mich aus einer praktischen Sicht und unter Berücksichtigung der Mehrsprachigkeit in den Medien in multilingualen Gesellschaften diesem Thema nähern. 
Förderung der Mehrsprachigkeit sowie der sprachlichen und kulturellen Vielfalt, das waren die zentralen Botschaften im „Jahr der Sprachen 2001“, zu denen der Europarat und die Europäische Union gemeinsam ausgerufen hatten. In diesem Sinne haben sich viele Autoren intensiv mit der Fragestellung auseinandergesetzt, welche Konsequenzen der Perspektivenwechsel hin zur Mehrsprachigkeit hat: Was verändert sich in den Medien unter dem Aspekt der Mehrsprachigkeit? Was die Entwicklung der Mehrsprachigkeit betrifft, schließe ich mich der Meinung von Schneider/Clalüna an, die bei der Untersuchung dieser Thematik zu folgender Feststellung kommen: "Der Baum der Mehrsprachigkeit - er ist nicht in den Himmel gewachsen, aber ein bisschen gewachsen ist er doch!“ (2002:2)

In unserem Zeitalter bilden die audiovisuellen Medien eine der bedeutendsten Säulen der Globalisierung. Die unterschiedlichen audiovisuellen Produkte werden den Menschen von den Managern der einflussreichen Kommunikations- und Informationskanäle (Telefonie, Internet, Zeitungen, Radio, Fernsehen) angeboten. Dabei ist das Ziel eine rasche weltweite Information und Teilhabe an der sozioökonomischen, kulturellen und politischen Entwicklung für alle. Neben dem Telefon haben sich seit den 1960er Jahren mit dem Mobiltelefon, dem Fax und dem Internet neue grenzenlose und immer rasantere Kommunikationstechnologien entwickelt. Vor allem über das Internet haben sich die grenzüberschreitenden Kommunikationsprozesse vervielfacht und die Zahl der Internetanschlüsse nimmt weiterhin weltweit exponentiell zu Die globalen kommerziellen Netzwerke (Berlusconi Medienkonzerne, Murdoch, Turner usw.) zeigen eine vereinheitlichende Tendenz bei ihren kulturellen Strategien sowie in der Sprachenverwendung, nämlich Englisch. Diese Tendenz könnte man als globalisierte Marketingstrategie bezeichnen. Die Zunahme von ethnisch und sprachlich spezifizierten (Minderheiten-)Medienprogrammen kann zugleich in regionalen Kontexten überall auf der Welt beobachtet werden. Diese Medienprogramme verleihen dem Sprachpluralismus Ausdruck. Sie wollen die Mehrsprachigkeit nicht zuletzt auch als Ressource von Vielfalt entwickeln und bewahren. Auf der Grundlage einer pragmatischen Einschätzung wird im vorliegenden Beitrag versucht, die Rolle des mehrsprachigen Medienangebots sowie dessen Grenzen $\mathrm{zu}$ analysieren. Konkrete Beispiele aus der algerischen Presse, die die Bedeutung von Sprachpluralität für den angestrebten soziokulturellen und ökonomischen Austausch in der nordafrikanischen Region hervorheben, werden aufgezeigt.

\section{Sprachpluralistische Medien und Globalisierung}

Der Erscheinung der Sprachen- und Kulturvielfalt in den einzelnen Ländern und Weltregionen wird bei dem Informationsprozess in den audiovisuellen Medien bewusst wesentliches Augenmerk gewidmet. Bei der thematischen Gestaltung der jeweiligen audiovisuellen Programme (Kultur, Wirtschaft, Politik, Religion, Sport, Liebe, Tourismus, Alltag usw.) versuchen die Medienproduzenten im Sinne einer höchstmöglichen Massenwirkung, die bekannten Weltsprachen (Englisch, Französisch, Arabisch, Spanisch, Deutsch, Chinesisch usw.) zu benutzen. Geleitet von dem Prinzip des Sprachenpluralismus werden dabei auch kulturelle Differenzen berücksichtigt. Inhaltlich müssen beispielsweise insbesondere im Bereich der Liebesfilme unterschiedliche Toleranzschwellen in unterschiedlichen Kulturregionen berücksichtigt werden. Aber auch aus dem Bereich des Produktmarketing weiß man, dass vielleicht bei Autos dasselbe Modell weltweit produziert und verkauft werden kann, in der Wahl des Produktnamens wird man sich aber den unterschiedlichen kulturellen Kontexten anpassen. Ein japanischer Autohersteller wählte vor 
einigen Jahren einen Autonamen, der in Frankreich wie „merde“ klingt, und konnte die Verkaufszahlen für dieses Modell auf dem französischen Markt völlig vergessen.

Um die ganze Bevölkerung und auch die hohe Anzahl von Analphabeten und Menschen, die ausschließlich ihre Muttersprache beherrschen, in Weltregionen außerhalb Zentraleuropas zu erreichen, werden in den verschiedenen Regionen Afrikas neben den weit verbreiteten Weltsprachen ebenfalls Sprachen wie Kiswahili, Luo, Wolof, Berberisch, Afrikaans in den Medien berücksichtigt. Ähnlich ist die Situation in Europa, wenn es sich um europäische Nationalsprachen wie z.B. Litauisch und Kroatisch handelt. Meist findet dies in so genannten Programmfenstern statt, die im Laufe des Tages eingeschaltet werden, so beispielsweise auch auf BBC World, und die in Afrika im Laufe des Tages regelmäßig 30 Minutenfenster in Landessprachen einschieben. Aber lokale Radiosender kennt man ja auch in den urbanen Zentren in Europa und in den USA, wo seit der Kommerzialisierung der audiovisuellen Medien immer mehr Stadtsender ihre Arbeit aufnehmen und seitdem es in den 1980er Jahren mehr Satelliten und mehr Frequenzen zu verteilen gibt. Teilweise verbreiten lokale Medien, die schwerer zu kontrollieren sind, politisch motivierte radikale Programme und senden Hassbotschaften, um Bevölkerungsgruppen gegeneinander aufzuhetzen. So existieren in den USA verschiedene xenophobe Sender. Gleiches gilt für Ruanda zu Zeiten des Krieges 1994 bis 1998, als lokale Sender öffentlich Mordaufrufe sendeten. Pluralität kann immer auch destruktiv eingesetzt werden.

Nun möchte ich zur Frage kommen, was Globalisierung mit Medien-Sprachenpluralität zu tun hat. Zunächst die positive Nachricht: Im Kontext der Globalisierung hat der Sprachenpluralismus in den Medien eine hohe Präsenz erreichen können. Dies ist keineswegs nur in den europäischen Ländern festzustellen, sondern auch in den verschiedenen Regionen Afrikas. Die schlechte Nachricht lautet aber: Der oben erwähnte positive und florierende Aspekt der demokratischen pluralistischen Medienpolitik, die weltweit Anerkennung findet, wird in vielen Ländern der so genannten Entwicklungs- und Schwellenländern aus willkürlichen Gründen nicht systematisch berücksichtigt. Während des Tunistreffens der UN zu Internetdomänen und Informationsgesellschaft (SMSI), das vom 16.-18. November 2005 stattfand, kamen die Teilnehmer zu der Feststellung, dass das Internet von politischen Einrichtungen durchaus kontrolliert bzw. zensiert wird (vgl. dazu die algerische Tageszeitung Le Quotidien d'Oran vom 15.11.2005). Die Kongressteilnehmer bzw. die Internetnutzer und Internetbetreiber scheinen noch viel Überzeugungsarbeit leisten zu müssen auf dem Weg zur freien weltweiten Mediennutzung.

Ein anderer wichtiger Aspekt ist die Frage der im Netz genutzten und nutzbaren Sprachen, die für die soziale, kulturelle und wirtschaftliche Entwicklung eine zentrale Bedeutung haben. 90\%der gut 6.000 Sprachen weltweit sind im Internet überhaupt nicht vertreten. Dadurch werden lokale Sprachen, Kulturen, und entsprechendes kulturspezifisches Wissen im Internet unsichtbar und in den Hintergrund verdrängt. Damit verfestigt sich auch eine monolineare eingeschränkte Weltsicht. In den Cybercafés dominiert Englisch. Daneben spielen asiatische und andere europäische Sprachen eine gewisse Rolle. Insgesamt elf Sprachen repräsentieren $90 \%$ der Netzinhalte. Allerdings sind $80 \%$ der im Netzangebot verfügbaren Inhalte in englischer Sprache verfasst (Merkel 2003:1). 


\section{Medienstrategien in mehrsprachigen Ländern}

Wenn wir die Schweiz oder Algerien als vielsprachige Länder bezeichnen, bedeutet das, dass Deutschland und Österreich einsprachige Länder sind? Auf den ersten Blick sicher ja - in Deutschland und in Österreich spricht man Deutsch. Die Bürger in diesen beiden deutschsprachigen Ländern haben zwar das Deutsche als gemeinsame Sprache, das von allen gesprochen und verstanden wird. Aber die eigentliche Muttersprache der Deutschen oder der Österreicher bzw. der großen Mehrheit der Bevölkerung in diesen beiden Ländern ist nicht das Hochdeutsche, sondern einer der vielen Dialekte. Diese Dialekte sind ja höchst unterschiedlich und einem Bayern oder Schwaben geht es sicher nicht anders, als es uns ausländischen Germanisten geht, wenn wir in Norddeutschland erstmals Plattdeutsch hören. Am Anfang meines mehrjährigen Leipzigaufenthalts benötigte ich einige Wochen, bis ich der Unterhaltung an bestimmten Orten, wo gesächselt wurde, wenigstens in groben Zügen folgen konnte. In diesem Sinne ist die Mediensituation in Deutschland nicht anders als im mehrsprachigen Algerien oder in der deutschsprachigen Schweiz, wo auch ganz unterschiedliche Dialekte gesprochen werden.

Mit den vier Sprachen (Algerisch, Arabisch, Französisch, Berberisch), die im Alltag bzw. auch im Journalismus benutzt werden, ist Algerien tatsächlich ein vielsprachiges Land, was allerdings nicht bedeutet, dass jeder einzelne Algerier alle diese vier Sprachen beherrscht. In der Tat ist es nur ein Teil der Bevölkerung, der praktisch über bilinguale Kompetenzen verfügt, d.h. wenigstens die beiden Hauptsprachen - Arabisch und Französisch - fließend beherrscht. Viersprachig ist wohl kaum jemand. Um eine solche, also viersprachige Sprachpluralität zu ermöglichen, bräuchte man eine Verständigung zwischen den genannten verschiedenen Sprachen, die auch unterschiedliche Kulturen und Mentalitäten repräsentieren. In den Medien erscheinen allerdings die wichtigsten Informationen in den vier am häufigsten verwendeten Sprachen: Algerisch, Hocharabisch, Französisch, Berberisch.

In Zeitungen, Radio und Fernsehen hat sich seit 1990 der Gebrauch des Algerischen immer mehr durchgesetzt, vor allem seit die Konkurrenz durch verschiedene Sender den Druck zu höheren Einschaltquoten durch mehr Publikumsnähe erhöht hat - ähnlich der kommerziellen Wende im Europa der 1980er Jahre. In den Nachrichtensendungen des lokalen algerischen Fernsehprogramms wird hingegen praktisch durchgehend Hocharabisch verwendet - zum einen, damit sich auch die Berberisch-, Französisch-, oder Algerisch-Sprechenden informieren können, zum anderen aber sicher auch, weil Hocharabisch mehr Seriosität suggeriert. Genauso verhält es sich in den arabischen Nachbarländern Tunesien, Marokko, Libyen, Mauretanien - sowie in anderen arabischen Staaten wie Ägypten, Syrien, Libanon, Saudi-Arabien. In allen diesen Ländern ebenso wie in Algerien existiert ein eigenes spezifisches arabisches Vokabular. Algerische Zeitungen verwenden z.B. ein anderes Vokabular bzw. andere Termini als die tunesischen und marokkanischen Zeitungen. Das Hocharabische ist der gemeinsame Nenner und ermöglicht erst eine interregionale Verständigung, auch wenn es mit vielen Bedeutungsunterschieden und vielfältigen Akzenten und regional eingefärbtem Wortschatz gesprochen wird. Dies ist ja auch in den deutschsprachigen Ländern der Fall. Während man in Deutschland z.B. den Begriff ,Fahrrad' verwendet, gebraucht man in der Schweiz ,Velo'. Um ein weiteres Beispiel anzuführen: Das deutsche ,Schreibwarengeschäft' heißt in der Schweiz ,Papeterie'. Kauft man in Deutschland eine ,Fahrkarte', benutzt man in der Schweiz ein ,Billet'. In schweizerischen Bahnhöfen findet man aber auch einen ,Fahrkartenschalter'. 


\section{Mehrsprachigkeit in den algerischen Medien}

Die algerischen Medien verfolgen, wie bereits erwähnt, das Prinzip der Sprachenpluralität, sowohl im Fernsehen und Rundfunk als auch in den Printmedien sowie im Internet. Nachfolgend möchte ich einen kurzen Überblick über die Entwicklung der Sprachpolitik bzw. der sprachpluralistischen Medienstrategie in Algerien geben.

Nach den Oktoberereignissen (1988) in Algerien ist das Medienfeld im Wesentlichen abgewickelt worden. Bis zu diesem Jahr waren die unterschiedlichen Medien zentralistisch organisiert. Lokale Dialekte sowie die berberische Sprache, die in verschiedenen Orten bzw. Städten Nord- und Südalgeriens z.B. Bejaïa, Tizi Ouzou, Ouargla, Ghardaïa, Tamanrasset, wo Berberisch täglichen gebraucht wird, waren im nationalen Medienpanorama nicht vertreten. Aufgrund der seit 1962 bzw. seit der Unabhängigkeit durchgeführten Arabisierungspolitik hatte Hocharabisch im algerischen Fernseh-, Radio- und Zeitungsprogramm den Vorrang. Französisch, das von einem großen Teil der algerischen Bevölkerung gesprochen wird und als Handels- und Verkehrssprache gilt - Algerien ist nach wie vor ein frankophones Land, wo Französisch im öffentlichen Leben starke Verbreitung findet - rückte somit in den Hintergrund. Dank der neuen sprachpolitischen Maßnahme, die darin besteht, sowohl den Gebrauch des Französischen als auch des Berberischen und der anderen regionalen Dialekte im Medienbereich zu ermöglichen, konnte sich der Sprachpluralismus in der algerischen Presselandschaft zunehmend durchsetzen. Somit konnte der Kommunikations- und Informationsprozess zwischen der Presse, den staatlichen Einrichtungen und dem Publikum wesentlich vereinfacht werden. Ab 1990 wurde Berberisch unter der Regierung von Ghozali in das staatliche Fernsehprogramm in Form einer Nachrichtensendung aufgenommen.

Im Jahr 1990 machte die Hamrouche-Regierung die geschriebene Presse dem privaten Sektor zugänglich. So erschienen in diesem Jahr auch die ersten privaten bzw. unabhängigen Tageszeitungen Liberté (Französisch), El Watan (Französisch), El Khabar (Arabisch). Im Hinblick auf die sprachpolitische Ebene wurde 2000 Berberisch (Tamazight) als nationale Sprache anerkannt. 2004 erschienen in den Zeitungen die ersten Seiten auf Tamazight.

In den algerischen Medien werden heute die drei Sprachen Arabisch, Französisch und Berberisch breit genutzt. Die regionalen Radiosender berücksichtigen die entsprechenden Dialekte. Der erste nationale Radiosender ,Chaîne une (1)' gestaltet sein Programm in arabischer Sprache. Der zweite nationale Radiosender, Chaine trois (3)' bietet sein ganzes Programm in französischer Sprache an. Der Oraner Radiosender, genannt ,El Bahia', der sowohl Arabisch, als auch Französisch sowie Algerisch und andere Dialekte berücksichtigt, genießt im ganzen Lande, inzwischen aber auch im Ausland, großes Ansehen. Dank des pragmatischen Charakters seiner Kommunikations- und Sprachstrategie wird er von einem großen Teil der Zuhörerschaft bevorzugt.

Bis auf die Nachrichten, die im algerischen Fernsehprogramm auch in berberischer Sprache gesendet werden, wird der Rest des TV-Programms von der ENTV (Entreprise Nationale de Télévision) in arabischer Sprache angeboten. Das zweite Fernsehprogramm, ,Algerian TV' oder auch ,Canal Algérie' genannt, benutzt hauptsächlich Französisch. Dort werden Nachrichten auch in englischer Sprache ausgestrahlt. Das dritte Fernsehprogramm ,Sat 3' präsentiert alle Sendungen in arabischer Sprache. Die beiden letzt genannten 
Fernsehprogramme sind ans internationale Satellitennetz angeschlossen und können weltweit empfangen werden.

Im Hinblick auf die Printmedien erscheinen die folgenden wichtigsten Tageszeitungen auf Arabisch: El Khabar, Echourouk, Eljaoum, Elahdath, Ennasr, Eljoumhouria, Sawt El Gharb, Manbar El Gharb, Sada Wahran, Echaab. Neben diesen Tageszeitungen werden z.B. folgende Wochenzeitungen in arabischer Sprache gedruckt: Adaou El Jawmi, Panorama, Erriadhi, Malaib, Sayidati. Folgende wichtige Tageszeitungen erscheinen in französischer Sprache: El Moudjahid, El Watan, Liberté, le Quotidien d'Oran, la Voix de l'Oranie, l'Echo d'Oran, Ouest Tribune, la Nouvelle République, le Jeune Indépendant, le Soir d'Algérie, Horizon, la Tribune. Zum Französischprogramm gehören auch Wochenzeitungen und Magazine wie Confidences. Mit einer Auflage von 350.000 Exemplaren steht die arabische Tageszeitung El Khabar an erster Stelle. An zweiter Stelle steht die französische Tageszeitung Le Quotidien d'Oran mit 198.000 Exemplaren.

Sowohl die nationale Tageszeitung El Moudjahid (Französisch und Berberisch mit lateinischen Buchstaben) als auch die nationalen Tageszeitungen Echaab (Arabisch und Berberisch mit arabischen Buchstaben) und Algérie News (Arabisch und Französisch) werden zweisprachig veröffentlicht.

Zu dem Programm der beiden Tageszeitungen (La Voix de l'Oranie und Sawt al Gharb), die zusammengehören, gehört das Projekt „Deutsche Seite“, das in absehbarer Zukunft in Form einer bilateralen Zusammenarbeit konkretisiert werden soll.

Was die Verteilung des Sprachgebrauchs anbelangt, so lässt sich festhalten, dass Arabisch dominant ist, sowohl in den Printmedien als auch im Fernsehen und im Rundfunk. Französisch steht nach wie vor an zweiter Position, während Berberisch an dritter Stelle steht.

\section{Mehrsprachigkeit und Übersetzung}

Ein großes Problem, vor dem die Journalisten bei der Übersetzung bestimmter Berichte beispielsweise vom Französischen ins Arabische und umgekehrt - stehen, ist die Äquivalenzproblematik. Aufgrund der Kulturunterschiede ist es häufig nicht einfach, einen gleichwertigen Begriff zu finden. Aus diesem Grund ergibt sich für algerische Journalisten die dringende Notwendigkeit, sowohl der Herkunftssprache als auch der Zielsprache mächtig zu sein, damit es nicht zu gravierenden Fehlinterpretationen und fehlleitenden Interferenzen kommt. Andauernd werden algerische Journalisten mit dem Phänomen der zunehmenden Terminologie konfrontiert. Aufgrund der Globalisierung wächst das Arbeitsfeld der Journalisten immer häufiger über Landes- und Sprachgrenzen hinaus. Berufstätige aus der Medienbranche benötigen bei der Suche nach der richtigen Übersetzung von Termini entsprechende zweisprachige Fachwörterbücher.

\section{Bibliographie}

Chlosta, C. u.a. 2001. Zum Erhalt und zur Förderung von Mehrsprachigkeit. In: E-Papiere zu Sprachwissenschaft und Sprachdidaktik, Elise Heft 1: 7-10.

Mefftah Tlili, N. 2003. Traduction et plurilinguisme / Traduction et culture. In: Traduire la langue traduire la culture. Sud Editions Maisonneuve \& Larose. pp. 169-177. 
Merkel, M.C. 2003. Gleichberechtigter Zugang zum Wissen und Stärkung der Meinungsfreiheit. Das UNESCO Kommunikationsprogramm 2004-2005. In: Unesco heute online Online-Magazin der Deutschen UNESCO-Kommission; Ausgaben 11-12, November / Dezember.

Müller, G. u.a. 2004. Medien Jargon - Handbuch für Journalisten im Ausland - Fachbegriffe ins Englische, Spanische und Französische übersetzt. G.r.u.p.p.e M.

Schneider, G. und M. Clalüna. 2002. Mehrsprachigkeit und Deutschunterricht. In: Bulletin vals-asla, Sonderheft. Université de Neuchâtel.

Seddiki, A. 2002. Semantische Äquivalenzprobleme bei der Übersetzung arabischer Texte ins Deutsche. In: Tradtec-Zeitschrift ,Übersetzung und Sprachen' 01/2002: 46-52, Oran.

Six, C. 2001. Hindu-Nationalismus und Globalisierung. Die zwei Gesichter Indiens: Symbole der Identität und des Anderen. Frankfurt a.M./Wien: Brandes \& Apsel/Südwind.

Wandeler, J. 2001. Comprenez-vous only Bahnhof? Mehrsprachigkeit in der Mediendokumentation. In: INFO 7, Heft 3 (12/2001), Zürich: 138-143.

\section{Biographical note}

Aoussine Seddiki was born 1955. He studied German at the University of Oran (Algeria) from 1975 until 1978 and received his Diplome des Etudes Approfondies in Methodology at the University of Algier in 1983. In 1992 he received his Ph.D. in German as a Foreign Language at the University of Leipzig. 1978 - 1982 he worked as teacher of German at various high schools in Algeria. Since 1981 he has been conducting research and teaching at the German section of the University of Oran, and since 2000 he is a university professor. He has authored numerous publications in academic journals and volumes. His research fields include German as foreign language, methodology, intercultural German studies, and translation studies. 